\title{
Adamantyl-functionalized polymer monolith for capillary electrochromatography
}

\author{
Kaname Ohyama ${ }^{\mathrm{a}, \mathrm{b} *}$, Yuki Fukahori ${ }^{\mathrm{a}}$, Kenichiro Nakashima ${ }^{\mathrm{c}}$, Tomoyuki Sueyoshi ${ }^{\mathrm{a}}$, \\ Naoya Kishikawa ${ }^{\mathrm{a}}$, Naotaka Kuroda ${ }^{\mathrm{a}}$
}

a Department of Environmental and Pharmaceutical Sciences, Graduate School of Biomedical Sciences, Nagasaki University, Nagasaki, 852-8521, Japan

${ }^{\mathrm{b}}$ Nagasaki University Strategy for Fostering Young Scientists, Nagasaki, 852-8521, Japan

${ }^{c}$ Department of Clinical Pharmacy, Graduate School of Biomedical Sciences, Nagasaki University, Nagasaki, 852-8521, Japan

* Corresponding author. Department of Environmental and Pharmaceutical Sciences, Course of Pharmaceutical Sciences, Graduate School of Biomedical Sciences, Nagasaki University, Nagasaki, Japan. Tel.\&Fax: +81-95-819-2446

E-mail address: k-ohyama@nagasaki-u.ac.jp

Keywords: adamantane / basic solute / capillary electrochromatography / peak tailing / polymer-based monolith 


\begin{abstract}
An adamantyl (ADM)-functionalized monolithic stationary phase was newly synthesized by a single-step copolymerization of 1-adamantyl-( $\alpha$ - trifluoromethyl) acry-late, ethylene dimethacrylate, and 2-acrylamido-2-methyl-1-propanesulfonic acid in order to prevent the peak tailing of basic solutes in capillary electrochromatography and was compared with butyl methacrylate (BMA)-based one. The ADM structure shields the negatively charged groups on the surface of monolith from basic solutes, resulting in better peak shapes than BMA-based monolithic stationary phase. As the monomers ratio decreased, the monolithic column had lower retention and higher column efficiency which was likely due to lower phase ratio and smaller globule size of monolith, respectively. The ADM-functionalized monolithic columns exhibited a good repeatability and reproducibility of column preparation with relative standard deviation values below $9 \%$ in the studied chromatographic parameters.
\end{abstract}




\section{Introduction}

Organic polymer-based monolithic columns have been successfully used in capillary electrochromatography (CEC) [1-8]. Polymer-based monoliths are attractive because there are a wide variety of different monomers available and can be used within a broad pH-rage where they are chemically stable. They are prepared by a single-step copolymerization with monomer, cross-linker, pore-forming solvent and initiator.

Different from the monolithic columns in high performance liquid chromatography (HPLC), the introduction of charged moiety into the monolithic network is essential in CEC for generation of electroosmotic flow (EOF). Consequently, moderately or strongly ionic monomers, i.e. 2-acrylamido-2-methyl-1-propanesulfonic acid (AMPS), [2-(methacryloyloxy)ethyl]-trimethylammonium chloride, are extensively utilized in the preparation of polymer monoliths for CEC [1-5]. In the CEC separation of charged solutes, these monoliths work as a mixed-mode stationary phase that combines two retentive interaction (i.e. hydrophobic and cationic-exchange or anion-exchange) $[9,10]$. However, CEC of charged solutes on such stationary phases often suffers from severe peak tailing due to Coulombic attraction between solutes and monolith surface of opposite charges.

In order to juggle production of stable EOF and prevention of peak tailing in CEC, new type of capillary monolithic columns have been developed. One type has a positively charged polymer layer on the inner wall of capillary and a neutral monolithic packing as the bulk stationary phase [11]. Another type is the shielded stationary phase which was prepared via photoinitiated grafting of a covering layer of hydrophobic 
polymer chain onto the ionizable surface of a porous monolith [12]. However, the preparation of these columns requires the multi-step procedure.

HPLC using the columns packed with silica-gel particles also suffers from peak tailing of basic solutes due to the interaction between solutes and silanols of packing material. In this context, some groups proposed the steric exclusion of the solute from the surface of silica-gel particles to reduce the interaction. Hemetsberger et al. observed that longer alkyl chains partially prevent solutes from reaching the underlying surface and play an important role in blocking the silanols [13]. Gilpin et al. and Guiochon et al. demonstrated improved peak symmetry for basic solutes by the bulky rigid structure of adamantane shielding the unreacted silanols from even small solutes $[14,15]$.

With the idea to use adamantane as a shield to reduce the peak tailing of charged solutes in CEC, we newly synthesized adamantyl (ADM)-functionalized polymer monolith by a single-step copolymerization with the monomer containing ADM structure and a cross-linker. In the present study, small basic solutes were used to evaluate the peak symmetry on ADM-functionalized and butyl methacrylate (BMA)-based monolithic columns. To use the ADM-functionalized monolithic column in CEC, some fundamental properties of it were also investigated. To the best of our knowledge, this is the first attempt to develop the polymer-based monolith by a single-step copolymerization for prevention of peak tailing of ionic analytes in CEC. 


\section{Materials and methods}

\subsection{Chemicals}

Butyl methacrylate (BMA), ethylene dimethacrylate (EDMA), 2-acrylamido-2-methyl-1-propanesulfonic acid (AMPS), $\alpha, \alpha$ '-azobisisobutyronitrile (AIBN), 1,4-butanediol and 3-(trimethoxysilyl)propyl methacrylate were obtained from Nacalai Tesque (Kyoto, Japan). 1-Adamantyl-( $\alpha$-trifluoromethyl)acrylate (MAF-ADE, Fig. 1) was kindly gifted from Tosoh Organic Chemical (Yamaguchi, Japan). 1-Propanol, acetic acid, sodium acetate, potassium dihydrogenphosphate, dipotassium hydrogenphosphate, aniline, $\quad N$-methylaniline, $\quad N, N$-dimethylaniline, benzene, naphthalene and anthracene were from Wako (Osaka, Japan). Thiourea was obtained from Kishida Chemical (Osaka). HPLC grade of acetonitrile (ACN) was purchased from Kanto Chemical (Tokyo, Japan).

\subsection{Instrumentation}

All the CEC experiments were performed on a CAPI-3200 system equipped with a photodiode array UV detector (Otsuka Electronics, Osaka). Fused-silica capillaries (375 $\mu \mathrm{m}$ o.d. x $75 \mu \mathrm{m}$ i.d.) were obtained from Polymicro Technologies (Phoenix, AZ, USA). Total, packed and effective lengths of capillary columns used here were 42.0 $\mathrm{cm}, 30.0 \mathrm{~cm}$ and $30.5 \mathrm{~cm}$, respectively. The mobile phase, prepared by mixing ACN and the buffer (phosphate or acetate buffer), was degassed thoroughly prior to use. At the beginning of each day's work, the capillary column was conditioned with a mobile phase for $1 \mathrm{~h}$ and equilibrated by applying voltage $1,5,10 \mathrm{kV}$ for $5 \mathrm{~min}$ each. Instead 
of pressuring at both ends of the capillary column, the CEC system was thermostatically maintained at $18{ }^{\circ} \mathrm{C}$ throughout the analysis in order to avoid bubble formation within the capillary column. The separation voltage was set at $10 \mathrm{kV}$ and the injections were made by applying a voltage of $10 \mathrm{kV}$ for $8 \mathrm{sec}$.

\subsection{Column preparation}

Monoliths were prepared by in situ polymerization in fused-silica capillary. To ensure covalent attachment of monolith to the inner capillary wall, surface modification of the wall was performed with 3-(trimethoxysilyl)propyl methacrylate prior to the polymerization reaction [16]. Monoliths were prepared by thermally initiated free-radical polymerization of the bulk monomer (MAF-ADE), a cross-linker (EDMA) and a monomer with a sulfonic acid group (AMPS) for the generation of EOF. A mixture of water, 1-propanol and 1,4-butanediol were used as pore-forming solvents following a recipe developed for BMA-based monolith $[1,4]$. Each material in a solution was bubbled with nitrogen for $20 \mathrm{~min}$ before mixing and after mixing, the mixture was sonicated for $10 \mathrm{~min}$. From one end, 50-cm long surface-modified capillaries were filled with the polymerization mixture up to a length of $45 \mathrm{~cm}$ and both ends were plugged with a piece of septum. Both the capillary and the vial containing the reminder mixture were kept at $60{ }^{\circ} \mathrm{C}$ in an oven for $20 \mathrm{~h}$. The resulting columns were flushed first with water-ACN mixture $(20 / 80, v / v)$ to remove pore-forming solvents and unreacted monomers and then with mobile phase by an HPLC pump. A detection window was made adjacent to the monolithic material by burning the 
polyimide coating away. The capillary columns were equilibrated by successively applying 1, 5, $10 \mathrm{kV}$ for $10 \mathrm{~min}$ each.

The polymer monolith in the vial was cut into small pieces and Soxhlet extracted with methanol for $20 \mathrm{~h}$ to remove any soluble compounds and then vacuum-dried at $60{ }^{\circ} \mathrm{C}$ for $6 \mathrm{~h}$. This polymer was used for the determination of pore size distribution by mercury-intrusion porosimeter (PoreMaster 60GT, Sysmex, Hyogo, Japan).

\section{Results and discussion}

The retention factor $(k)$ was determined by:

$k=\left(t_{\mathrm{r}}-t_{\mathrm{o}}\right) / t_{\mathrm{o}}$

in which $t_{\mathrm{r}}$ and $t_{\mathrm{o}}$ were retention time of analytes and an EOF marker, respectively.

The EOF mobility $\left(\mu_{\mathrm{EOF}}\right)$ was calculated by:

$\mu_{\mathrm{EOF}}=u / E$

where $E=10 \mathrm{kV} / 420 \mathrm{~mm} ; u=300 \mathrm{~mm} / t_{0}$.

A measure of the symmetry of a peak, given by the following equation:

$S=W_{0.05} / 2 f$

where $S$ is symmetry factor, $W_{0.05}$ is the peak width at $5 \%$ height and $f$ is the distance from peak front to apex point at 5\% height.

The column efficiency $(N)$ was calculated from the number of theoretical plates per meter:

$N=5.55 \times\left(t_{\mathrm{r}} / W_{0.5}\right)^{2}$

where $W_{0.5}$ is peak width at half height. 
Height equivalent to a theoretical plate $(H)$ was determined by:

$H=L / N$

where $L$ is the capillary length packed with monolithic material (i.e. $300 \mathrm{~mm}$ ).

\subsection{Chromatographic property of ADM-functionalized monolithic stationary phase}

Chromatographic characterization was performed using aromatic hydrocarbons as a neutral model compound and thiourea as an EOF marker. A typical electrochromatogram of a separation of a mixture (benzene, naphthalene and anthracene) at $10 \mathrm{kV}$ is shown in Fig. 2. Based on the hydrophobic nature of adamantane, the elution order of model compounds were according to their hydrophobicity, indicating reversed-phase mode retention. The EOF mobilities $\left(\times 10^{-8}\right.$ $\mathrm{mm} / \mathrm{Vs}$ ) on the ADM-functionalized and BMA-based monolithic columns were 4.79 and 3.83, respectively. The ADM groups shielding of sulfonic acid groups never brings about a decrease in EOF.

\subsection{Peak shapes of basic compounds}

Using a mobile phase of $5 \mathrm{mM}$ acetate buffer ( $\mathrm{pH} 4.0)$ and $\mathrm{ACN}$ mixture, the test solutes (aniline, $N$-methylaniline and $N, N$-dimethylaniline) were analyzed on ADM-functionalized and BMA-based monolithic columns made from the polymerization mixture consisting 23.6\% MAF-ADE or BMA, 16.0\% EDMA, 0.4\% AMPS, 6.0\% water, 35.1\% 1-propanol and 18.9\% 1,4-butanediol. Table 1 shows retention factor and symmetry factor for the test solutes on both columns. 
On BMA-based monolithic column, the peaks were apparently tailing. The solutes $\left(\mathrm{p} K_{\mathrm{a}} 4.6 \sim 5.1[17]\right)$ are partially or fully positively charged, but they eluted slower than thiourea, which indicates the stronger effect of the interaction between the basic solutes and stationary phase than that of the electrophoretic mobility. Increased peak asymmetry on BMA-based monolith was observed in accordance with the increased basicity of the solutes. Elevating $\mathrm{ACN}$ content from 80 to $85 \%(\mathrm{v} / \mathrm{v})$ increased symmetry factor of the peaks. From these results, the poor peak shapes are most likely due to the binding of solutes to the sulfonic acid groups of the monolithic stationary phase.

On the other hand, the peak shapes were obviously better on ADM-functionalized monolithic column. The peaks of test solutes eluted slower than thiourea, indicating that the CEC separation is still mainly based on a chromatographic partition rather than electrophoretic principles. The better peak shapes on ADM-functionalized monolithic column should be due to the reduction of the accessibility of basic solutes to sulfonic acid groups. This shielding effect of adamantane observed in polymer-based monolith conforms with that in the ADM-modified silica used for HPLC [18]. Additionally, as shown in Table 1, the retention factor for basic solutes on ADM-functionalized monolithic column were lower than those on BMA-based one under the mobile phase conditions. This also supports the existence of the shielding effects which provides a decrease in Coulombic interaction between basic solutes and solfonic acid groups. 


\subsection{Influence of the composition of the polymerization mixture}

The morphology of monoliths is a direct consequence of the ratio between the monomer and pore-forming solvent phases, which is closely related to their chromatographic performance $[4,19]$. In this study, three monolithic stationary phases were prepared with different ratio between the monomer and pore-forming solvent phases in order to evaluate the influence of the ratio on retention and efficiency. The high-density polymer monolith was prepared with $40 \mathrm{wt} \%$ monomers and $60 \mathrm{wt} \%$ pore-forming solvents in the mixture. The medium- and low-density ones were prepared with monomers to pore-forming solvents ratios of 30:70 and 20:80, respectively. During this experiment, the content of AMPS, the ratio of MAF-ADE and EDMA and the composition of pore-forming solvents were kept constant.

For BMA-based monolith, it was reported that while a unimodal pore size distribution was observed for high-density material, pore size of low-density material was much broader and in some cases truly bimodal [4]. The broad pore size distribution of low-density material might lead to low column-to-column reproducibility of preparation. In our experiments, contrastingly, a unimodal distribution was obtained on all the ADM-functionalized monoliths including low-density monolith (Fig. 3). The preparation conditions (i.e. the ratio of monomers to pore-forming solvents, the composition of pore-forming solvents and polymerization temperature) for each monolithic material are similar to those of BMA-based monolith $[4,20]$; therefore, the difference in the pore size distribution could be attributed to the nature of MAF-ADE. Large pore size (around $5000 \mathrm{~nm}$ ) of ADM-functionalized monoliths was obtained, 
while the pore size of high density BMA monolith prepared in this study was $1030 \mathrm{~nm}$ (Fig. 3). The timing of the phase separation in the polymerization process determines the pore size and in general, earlier phase separation provides larger macropores. Therefore, the larger pore size of ADM-functionalized monolith seemed to be due to early phase separation.

As the density was decreased by decreasing the contents of monomers, the $k$ values of aromatic hydrocarbons became lower due to a decrease in the phase ratio. This is consistent with the previous study using BMA-based stationary phase [20]. Mediumand low-density monoliths were found to have 2-fold and 3-fold higher column efficiency, respectively, in comparison to high-density one. The pore sizes of three monoliths were within similar range from $4290 \mathrm{~nm}$ to $5560 \mathrm{~nm}$; therefore, the higher efficiency on lower density monolithic column should be attributed to the smaller globule size [4]. In the present state, ADM-functionaliozed monolithic columns exhibited low efficiency for benzene $(k=0.45,19600 \mathrm{~N} / \mathrm{m})$, naphthalene $(k=0.80$, $9600 \mathrm{~N} / \mathrm{m})$ and anthracene $(k=1.45,4300 \mathrm{~N} / \mathrm{m})$. On the other hand, the efficiency on BMA-based monolithic columns prepared in this study for benzene $(k=0.43,29300$ $\mathrm{N} / \mathrm{m})$, naphthalene $(k=0.79,9600 \mathrm{~N} / \mathrm{m})$ and anthracene $(k=1.37,2800 \mathrm{~N} / \mathrm{m})$ is also lower than those in the other literatures $[1,4,20]$. This means that the low efficiency on ADM-functionalozed monolithic columns was not due to the MAF-ADE monomer but due to unknown reason in our preparation procedure. Hence, the effect of degassing method in column preparation on improving the efficiency is now under the examination. 


\subsection{Effect of mobile phase composition and buffer concentration}

The effect of the ACN content in the mobile phase on the retention factors of the aromatic hydrocarbons was studied, using $5 \mathrm{mM}$ phosphate buffer $(\mathrm{pH} \mathrm{7.0)}$. The retention decreased with an increase in $\mathrm{ACN}$ content, in accordance with a reversed-phase mechanism. A linear relationship between log $k$ versus ACN content was obtained and the slopes were almost equal for all the different hydrocarbons. No significant change in EOF mobility was observed with the change in ACN content at a constant buffer concentration.

The effect of buffer concentration on EOF mobility in the range of 5-15 $\mathrm{mM}$ was examined with the mobile phase of phosphate buffer $(\mathrm{pH} \mathrm{7.0)}$ ) and $80 \%$ ACN. At the ionic strength used in this experiment, double-layer overlap which results in a decrease in the EOF mobility was expected to be negligible for the pores with $\mu$-meter size. A constant EOF mobility across ionic concentration range investigated was observed.

\subsection{Repeatability and reproducibility of monolithic column}

There are a few data on the reproducibility of preparation of acrylate monolithic columns in the literatures [20-24]. In accordance with previous work [21], the repeatability and reproducibility of preparation of ADM-functionalized monoliths were studied with five monolithic columns synthesized from a polymerization mixture (10.0\% MAF-ADE, 10.0\% EDMA, 0.4\% AMPS, 8.0\% water, 53.6\% 1-propanol, 18.0\% 1,4-butanediol). The three columns (No.1-3) were prepared from one polymerization mixture and the others (No.4-5) were prepared from another polymerization mixture but 
both of the same composition. The repeatability and reproducibility were expressed as relative standard deviations (RSD) of the chromatographic parameters, i.e. EOF mobility, retention times, retention factors and plate height, measured with the same set of aromatic hydrocarbons (Table 2). The run-to-run repeatability was evaluated with the No. 1 column for six measurements. The column-to-column repeatability was calculated within the first set of three columns (No. 1-3). The run-to-run repeatability was lower than $6 \%$ and the column-to-column one was below $8 \%$ for EOF mobility and all other parameters about the aromatic hydrocarbons. The reproducibility of five columns was also less than $9 \%$ for all the parameters. The repeatability and reproducibility obtained here permits the application of ADM-functionalized monolith for practical use in CEC.

\section{Conclusion}

It is demonstrated that CEC with ADM-functionalized monolithic stationary phase can provide good peak shape of basic solutes. The chromatographic retention was found to be based on a mixed-mode retention mechanism (hydrophobic interaction and Coulombic interaction). However, Coulombic interaction for positively charged solutes should be attenuated compared with BMA-based monolithic column by the shielding effect of adamantyl groups. Preparing three monolithic columns with different ratio between the monomer and pore-forming solvent phases, the monolith with lower density of monomers was observed to give lower retention and higher efficiency. For low-density monolithic column, satisfactory run-to-run and 
column-to-column repeatability and mixture-to-mixture reproducibility for electrochromatographic parameters were observed. Since the mechanism of preventing the peak tailing is likely based on shielding of the ionic groups on monolith surface from ionic solutes, ADM-functionalized monolith will be useful for CEC separation of acidic and basic solutes in both of anodic and cathodic EOF modes.

\section{Acknowledgement}

K. O. is grateful to the support by Special Coordination Funds for Promoting Science and Technology of the Ministry of Education, Culture, Sports, Science and Technology (MEXT). The authors thank Tosoh Organic Chemical for kindly supplying MAF-ADE monomer and also gratefully acknowledge the technical support on the measurement of pore size distribution by Mr. Masahide Takase (Daiso Co., LTD, Osaka). 


\section{References}

[1] E.C. Peters, M. Petro, F. Svec, J.M.J. Fréchet, Anal. Chem. 70 (1998) 2288.

[2] F. Švec, J.M.J. Fréchet, in: F. Švec, T.B. Tennikova, Z. Deyl (Eds.), Monolithic Materials, Elsevier, Amsterdam, 2003, pp. 20.

[3] T. Jiang, J. Jiskra, H.A. Claessens, C.A. Cramers, J. Chromatogr. A 923 (2001) 215.

[4] S. Eeltink, J.M. Herrero-Martinez, G.P. Rozing, P. J. Schoenmakers, W. Th. Kok, Anal. Chem. 77 (2005) 7342.

[5] B. L. Waguespack, S.A. Hodges, M.E. Bush, L.J. Sondergeld, M.M. Bushey, J. Chromatogr. A 1078 (2005) 171.

[6] P. Pruim, M. Öhman, Y. Huo, P.J. Schoenmakers, W. Th. Kok, J. Chromatogr. A $1208(2008) 109$.

[7] X. Wang, H. Lü, X. Lin, Z. Xie, J. Chromatogr. A 1190 (2008) 365.

[8] X. Wang, X. Lin, Z. Xie, J.P. Giesy, J. Chromatogr. A 1216 (2009) 4611.

[9] K. Ohyama, N. Kuroda, J. Liq. Chromatogr. \& Rel. Technol. 30 (2007) 833.

[10] X. Yang, J. Dai, P.W. Carr, J. Chromatogr. A 996 (2003) 13.

[11] Y. Li, R. Xiang, C. Horváth, J.A. Wilkins, Electrophoresis 25 (2004) 545.

[12] E.F. Hilder, F. Svec, J.M.J. Fréchet, Anal. Chem. 76 (2004) 3887.

[13] H. Hemetsberger, W. Maasfeld, H. Ricken, Chromatographia 7 (1976) 303.

[14] S.S. Yang, R.K. Gilpin, Anal. Chem. 59 (1987) 2750.

[15] M.T. Aubel, J.J. Pesek, G. Guichon, J. Chromatogr. 514 (1990) 11.

[16] C. Yu, M.N. Davey, F. Svec, J.M.J. Fréchet, Anal. Chem. 73 (2001) 5088.

[17] SciFinder, The Values Calculated Using Advanced Chemistry Development (ACD) 
Software Solaris, American Chemical Society, Washington, DC, 2007.

[18] M.T. Matyska, J. J. Pesek, S. Tong, J.E. Sandoval, J. Liq. Chromatogr. \& Rel. Technol. 26 (2003) 1169.

[19] F. Svec, J. Sep. Sci. 27 (2004) 747.

[20] S. Eeltink, G.P. Rozing, P.J. Schoenmakers, W. Th. Kok, J. Chromatogr. A 1109 (2006) 74 .

[21] J. Grafnetter, P. Coufal, E. Tesařová, J. Suchánková, Z. Bosáková, J. Ševčík, J. Chromatogr. A 1049 (2004) 43.

[22] V. Augustin, A. Jardy, P. Gareil, M.-C. Hennion, J. Chromatogr. A 1119 (2006) 80.

[23] A. Cantó-Mirapeix, J.M. Herrero-Martínez, C. Mongay-Fernández, E.F. Simó-Alfonso, Electrophoresis 29 (2008) 3866.

[24] A. Cantó-Mirapeix, J.M. Herrero-Martínez, C. Mongay-Fernández, E.F. Simó-Alfonso, Electrophoresis 29 (2008) 4399. 


\section{Figure captions}

Fig. 1 Structure of 1-adamantyl-( $\alpha$-trifluoromethyl)acrylate (MAF-ADE).

Fig. 2 CEC separation of aromatic hydrocarbons on ADM-functionalized monolithic column. Monolith (wt $\%$ ): 23.9\% MAF-ADE, 15.9\% EDMA, 0.4\% AMPS, 6.0\% water, 35.0\% 1-propanol, 18.8\% 1,4-butanediol. CEC conditions: mobile phase, $5 \mathrm{mM}$ phosphate buffer $(\mathrm{pH} 7.0) / \mathrm{ACN}=20 / 80$, v/v; voltage, $10 \mathrm{kV}$; injection, $10 \mathrm{kV}$ for $8 \mathrm{~s}$; UV detection, 210 and $250 \mathrm{~nm}$. Peaks: 1, thiourea; 2, benzene; 3, naphthalene; 4, anthracene.

Fig. 3 Pore size distributions of ADM-functionalized and BMA-based monoliths as obtained with different ratio of monomers and pore-forming solvents. High-density monolith (wt\%): 23.9\% MAF-ADE, 15.9\% EDMA, 0.4\% AMPS, 6.0\% water, 35.0\% 1-propanol, 18.8\% 1,4-butanediol. Medium-density monolith (wt\%): $18.0 \%$ MAF-ADE, $12.0 \%$ EDMA, $0.4 \%$ AMPS, 7\% water, $40.7 \%$ 1-propanol, $21.9 \%$ 1,4-butanediol. Low-density monolith (wt\%): 12.0\% MAF-ADE, 8.0\% EDMA, 0.4\% AMPS, 8\% water, 46.6\% 1-propanol, 25.0\% 1,4-butanediol. 
Table 1 Peak shapes of basic solutes on ADM-functionalized and BMA-based monolithic columns ${ }^{\mathrm{c})}$

\begin{tabular}{|c|c|c|c|c|c|c|c|c|c|}
\hline & \multicolumn{3}{|c|}{ Aniline } & \multicolumn{3}{|c|}{$N$-Methylaniline } & \multicolumn{3}{|c|}{$N, N$-Dimethylaniline } \\
\hline & $\mathrm{RT}$ (min) & $k$ & $S$ & $\mathrm{RT}$ (min) & $k$ & $S$ & $\mathrm{RT}$ (min) & $k$ & $S$ \\
\hline ADM-functionalized monolith ${ }^{\text {a) }}$ & 7.04 & 0.29 & 1.1 & 7.46 & 0.50 & 1.2 & 7.92 & 0.76 & 1.3 \\
\hline BMA-based monolith $^{\text {a) }}$ & 9.60 & 0.44 & 1.2 & 11.7 & 0.79 & 2.4 & 13.2 & 1.10 & 2.7 \\
\hline BMA-based monolith ${ }^{\text {b) }}$ & 9.40 & 0.36 & 1.3 & 12.6 & 0.76 & 3.9 & 14.4 & 0.96 & 5.8 \\
\hline
\end{tabular}

\footnotetext{
a) $5 \mathrm{mM}$ acetate buffer $(\mathrm{pH} 4.0) /$ acetonitrile $=20 / 80(\mathrm{v} / \mathrm{v})$

b) $5 \mathrm{mM}$ acetate buffer $(\mathrm{pH} 4.0) /$ acetonitrile $=15 / 85(\mathrm{v} / \mathrm{v})$

c) Monolith (wt \%): 23.6\% MAF-ADE, 16.0\% EDMA, 0.4\% AMPS, 6.0\% water, 35.1\% 1-propanol, 18.9\% 1,4-butanediol.

Separation condition: voltage, $10 \mathrm{kV}$; injection, $10 \mathrm{kV}$ for $8 \mathrm{~s}$; UV detection, $210 \mathrm{~nm}$. Other conditions as mentioned in Sec. 2.2.
} 
Table 2 Repeatability and reproducibility of preparation of ADM-functionalized monolithic columns expressed as RSD \% of EOF mobility, retention time, retention factor and plate height ${ }^{\mathrm{a}}$

\begin{tabular}{|c|c|c|c|}
\hline \multirow[t]{3}{*}{ Parameter } & \multicolumn{2}{|c|}{ Repeatability (RSD \%) } & \multirow{2}{*}{$\frac{\text { Reproducibility (RSD \%) }}{\text { Mixture-to-mixture }}$} \\
\hline & Run-to-run & Column-to-column & \\
\hline & Column No.1 (n=6) & Columns No.1-3 & Columns No.1-5 \\
\hline$\mu_{\mathrm{EOF}}$ & $\leq 1.8$ & $\leq 3.5$ & $\leq 3.4$ \\
\hline$t_{\mathrm{r}}$ & $\leq 2.5$ & $\leq 3.6$ & $\leq 3.9$ \\
\hline$k$ & $\leq 2.8$ & $\leq 2.4$ & $\leq 5.2$ \\
\hline$H$ & $\leq 5.9$ & $\leq 7.5$ & $\leq 8.5$ \\
\hline
\end{tabular}


Fig. 1

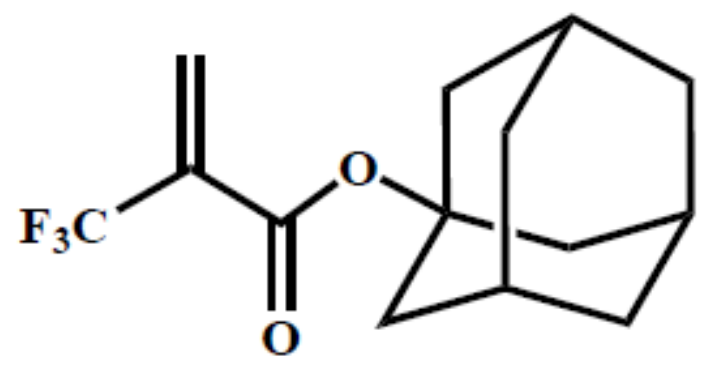

1-adamantyl-( $\alpha$-trifluoromethyl)acrylate 
Fig. 2

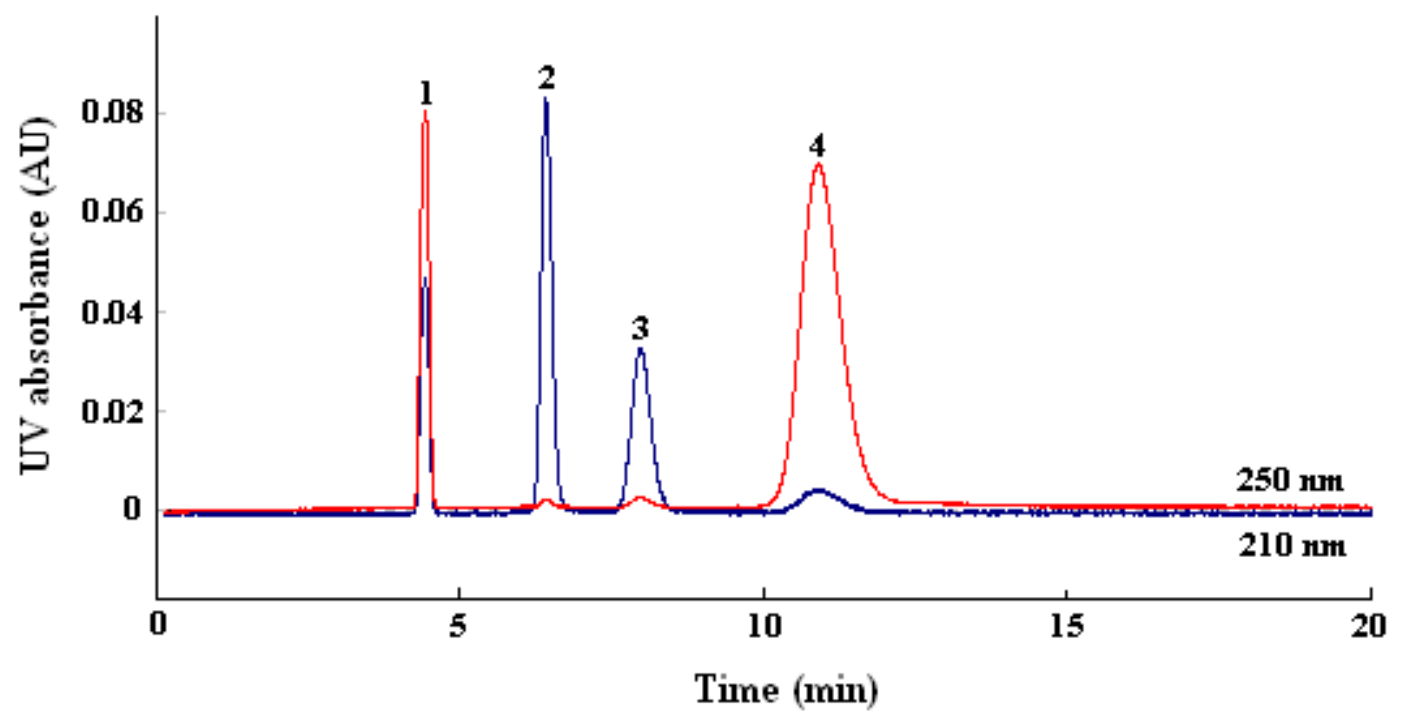


Fig. 3

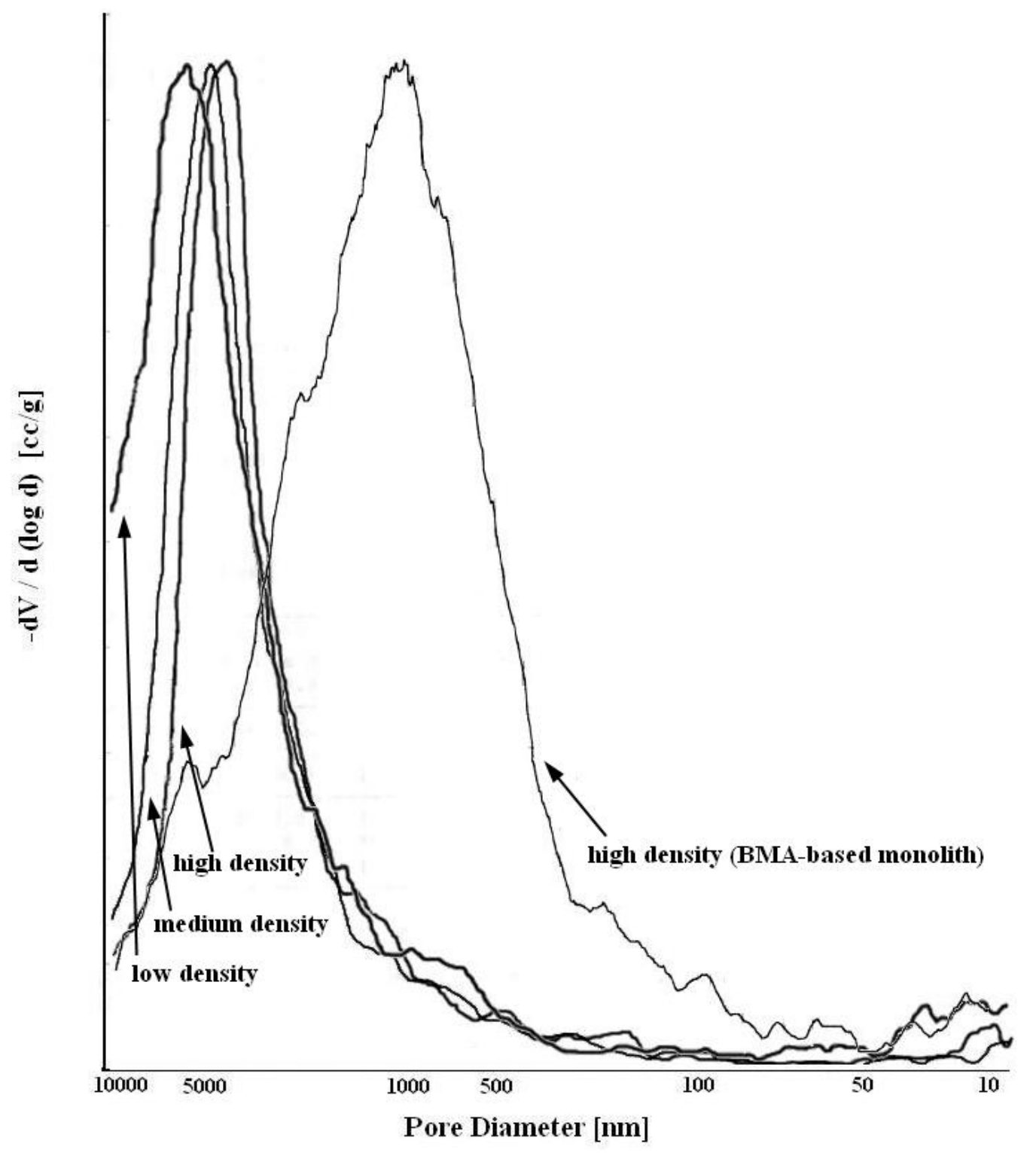

